\title{
Isotyping and Semi-Quantitation of Monkey Anti-Drug Antibodies by Immunocapture Liquid Chromatography-Mass Spectrometry
}

\author{
Xiaoxiao Huang, ${ }^{1}$ Xiaobin Xu, ${ }^{1,3}$ (i) Michael A. Partridge, ${ }^{2}$ Jihua Chen, ${ }^{2}$ Ellen Koehler-Stec, ${ }^{2}$ Giane Sumner, ${ }^{2}$ \\ Haibo Qiu, ${ }^{1,3}$ Albert Torri, ${ }^{2}$ and Ning $\mathbf{L i}^{1}$
}

Received 16 September 2020; accepted 23 November 2020; published online 6 January 2021

Abstract. There is an urgent demand to develop new technologies to characterize
immunogenicity to biotherapeutics. Here, we developed an immunocapture LC-MS assay to
isotype and semi-quantify monkey anti-drug antibodies (ADAs) to fully human monoclonal
antibody (mAb) drugs. ADAs were isolated from serum samples using an immunocapture
step with the Fab of the full-length mAb cross-linked to magnetic beads to minimize matrix
interference. A positive monoclonal antibody control against the human immunoglobulin
kappa light chain was used as a calibration standard for ADA quantitation. The final LC-MS
method contains 17 multiple reaction monitoring (MRM) transitions and an optimized 15-
min LC method. The results suggested that IgG1 was the most abundant isotype in ADA-
positive samples. IgG2 and IgG4 were identified at lower levels, whereas IgG3 and IgA levels
were only observed at very minor levels. In addition, levels of total ADA measured by the
LC-MS assay were comparable to results obtained using a traditional ligand binding assay
(LBA). The LC-MS ADA assay enabled rapid immunogenicity assessment with additional
isotype information that LBAs cannot provide.

KEY WORDS: ADA; immunogenicity; isotyping; LC-MS; monoclonal antibody.

\section{INTRODUCTION}

Biotherapeutics, such as monoclonal antibodies (mAbs), have been rapidly growing in the pharmaceutical market. However, these therapeutics have the potential to elicit an unwanted immune response that can result in the production of anti-drug antibodies (ADAs) (1-3). The binding of ADAs to a biological product can decrease the half-life of the product and/or have neutralizing activity. ADAs can therefore potentially reduce drug efficacy and pose concerns for patient safety.

A study of 121 US Food and Drug Administration (FDA)-approved biological products revealed that $89 \%$ of products showed ADA incidence, with almost half reporting some impact on efficacy, although only $26 \%$ reported impacts on PK (4). The generation of ADAs can be caused by product- or process-related factors such as the molecular structure of the biotherapeutic, product purity, posttranslational modifications, dose, route, or frequency of

\footnotetext{
${ }^{1}$ Analytical Chemistry, Regeneron Pharmaceuticals Inc., Tarrytown, New York 10591, USA.

${ }^{2}$ Bioanalytical Sciences, Regeneron Pharmaceuticals Inc., Tarrytown, New York 10591, USA.

${ }^{3}$ To whom correspondence should be addressed. (xiaobin.xu@regeneron.com; haibo.qiu@regeneron.com)
}

administration $(5,6)$. ADAs can also be induced by patientrelated factors including genetic profile, immune status, or disease status (7).

Although immunogenicity in animal models is not predictive of immunogenicity in humans, nonclinical immunogenicity studies are still informative for the evaluation of the impact of ADAs on pharmacokinetics and drug safety (8). Humans have five immunoglobulin isotypes including IgM, $\mathrm{IgG}, \mathrm{IgA}, \mathrm{IgE}$, and $\mathrm{IgD}$. Among them, IgM responds to the initial exposure of antigens, normally appearing within 7 days. $\mathrm{IgG}$ is the most abundant antibody and responsible for the long-term immune response to antigens. IgG has 4 subclasses including $\operatorname{IgG} 1, \operatorname{IgG} 2$, IgG3, and $\operatorname{IgG} 4$. IgA is mostly secreted into mucus, tears, and saliva. $\operatorname{IgE}$ is the least abundant isotype but it plays critical roles in allergic reactions and antiparasitic activity. IgD is mainly expressed on the surface of immature B cells (9). Similar to humans, monkeys also have five immunoglobulin isotypes, which include $\operatorname{IgM}, \operatorname{IgG}, \operatorname{IgA}, \operatorname{IgE}$, and $\operatorname{IgD}(10)$.

Detection and analysis of ADA formation are important for understanding immunogenicity and patient immune response in biological therapeutics development (11). Thus, the impact of ADA on product safety and efficacy of biological therapeutics should be evaluated during development. Additional immunogenicity assessments, such as isotyping, epitope mapping, and cross-reactivity may also be required by regulators. Commonly used methods for ADA 
detection are ligand binding assays (LBAs) using an electrochemiluminescence (ECL)-based bridging format $(7,12)$. In these assays, ADA forms a bridge between drugs labeled with two different tags or haptens, and these immune complexes are captured on a plate resulting in a signal in the assay. Although LBA provides high throughput, high sensitivity, and good specificity, interference from drugs, targets, and other serum components might cause false-positive or false-negative results. In addition, these methods may not be able to detect low abundant ADAs. Importantly, singleplex ligand binding assays are not capable of ADA isotyping or quantitation.

Surface plasmon resonance (SPR) is another widely used method to measure drug and ADA interaction in "real-time" (12), where serum samples flow through a drug-immobilized sensor chip and the changes in the refractive index are detected as a result of drug-ADA interactions. SPR can detect low-affinity antibodies for early immune response and enable isotyping and kinetics analysis. However, SPR has low throughput and low sensitivity.

With the rapid development of new instrumentation and methods, liquid chromatography-mass spectrometry (LC-MS) has emerged as a critical tool for the characterization of biologicals (13-15). Many efforts have been made to adopt the LC-MS-based approach as a supplementary tool for ADA analysis (16). One study used a bead-based immunoprecipitation method followed by LC-MS to assess ADA to human growth hormone analog (hGHA) (17) in order to overcome drug interference issues that are associated with conventional ADA assays. In this study, an excess amount of drug was used to saturate ADA binding sites. The drug-ADA complex was then captured by protein $G$ conjugated magnetic beads, followed by elution and digestion with cyanogen bromide $(\mathrm{CNBr})$. The $\mathrm{CNBr}-$ released drug peptides were quantified by LC-MS, enabling indirect quantitation of total ADAs.

In another study, bead extraction and acid dissociation (BEAD) were paired with LC-MS to simultaneously quantify the residual $\mathrm{mAb}$ drug, endogenous human $\mathrm{IgG}$, and the neutralizing antibody (NAb) (18). This approach efficiently removed high concentration drug and serum components, which can interfere in different analytical methods, and demonstrated the advantage of LC-MS in selectivity and multiplexing, providing a fast and direct approach to characterize multiple components for an immunogenicity assessment.

More recently, an immunocapture LC-MS method was developed for simultaneously isotyping and semi-quantitating pre-existing ADAs in human plasma (19). In this method, two strategies were used for ADA immunocapture. The first one used biotinylated drugs as the capture reagent to pull down ADAs. The second one took advantage of biotinylated antidrug $\mathrm{mAb}$ to form $\mathrm{mAb}$-drug-ADA complexes. The captured ADAs were immobilized on streptavidin magnetic beads so that they could be separated from human plasma. The eluted ADAs were digested and followed by LC-MS detection of specific universal peptides for each ADA isotype, demonstrating the ability of the immunocapture LC-MS method to detect pre-existing ADA in human plasma.

Subsequently, a similar immunocapture LC-MS method was developed for ADA identification in cynomolgus monkey plasma (20). Roos and his colleagues used a biotinylated mouse anti-drug mAb to capture ADA-drug complexes and isolated them by streptavidin magnetic beads. A common peptide for monkey IgG1-4 subclasses was used to detect the isolated ADAs. Shortly after, the same group developed a sequential immunoaffinity LC-MS assay to quantify therapeutic proteins in monkey plasma (21). For the first immunocapture step, a biotinylated mouse anti-drug antibody was used to capture the therapeutic protein in plasma. Following tryptic digestion, a unique peptide from the therapeutic protein was captured by the second immunocapture step using a mouse anti-peptide antibody, followed by LC-MS analysis. The sequential immunocapture steps can almost completely remove the matrix interference, providing a highly sensitive method to detect low abundant ADA in plasma samples. In addition, the use of an automated magnetic bead handler enables high throughput analysis.

Another study used blue native polyacrylamide gel electrophoresis (BN-PAGE) to separate human antibodyspecific immune complexes from unbound therapeutic antibodies and endogenous antibodies in monkey serum samples. The target fractions were cut from the gel and following in-gel digestion and LC-MS enabled a semi-quantitative analysis of humanized antibodies that were captured by ADAs (22).

In this study, we developed an immunocapture LC-MS method for ADA isotyping and semi-quantitation in serum samples from cynomolgus monkeys administered fully human mAb therapeutics. An ADA-positive control (ADA-PC) was used for assay development and to establish a calibration curve for semi-quantitation. The immunocapture procedure was optimized to maximize the recovery rate and minimize the nonspecific interaction with serum components. The captured ADAs were digested and analyzed by multiple reaction monitoring (MRM)-based LC-MS method. The method was successfully applied to isotype and semiquantify ADAs in monkey serum samples from single-dose and multiple-dose nonclinical studies. The total ADA responses measured by the LC-MS assay were consistent with responses obtained with a conventional LBA. In this study, the ADA-PC spiked-in monkey sera used to generate the calibration curve went through the same immunocapture LCMS procedure as the nonclinical samples, which we believe is the first reported effort of LC-MS-based ADA semi-quantitation. Comparing with previous efforts, the immunocapture optimization significantly increased assay sensitivity and reliability, allowing the LC-MS-based assay to obtain similar detection limits with traditional LBA.

\section{EXPERIMENTAL SECTION}

\section{Reagents and Chemicals}

The therapeutic drug candidate molecules, REGN10446P2 and REGN7798N, referred to hereafter as REGN-P and REGN-N, respectively, are fully human IgG4 mAbs. The therapeutic drug candidate molecules, REGN316P and REGN3592P3, referred to hereafter as REGN-6P and REGN-2P, respectively, are fully human IgG1 mAbs. REGN-ADA-PC, referred to hereafter as ADA-PC, is a monkey IgG1 mAb against the human kappa light chain. These antibodies were generated by Regeneron Pharmaceuticals, Inc. (Tarrytown, NY). Purified monkey IgA, 
$\mathrm{IgG}$, and $\mathrm{IgM}$ were purchased from Life Diagnostics, Inc. (West Chester, PA). Cynomolgus monkey sera were obtained from BioIVT (Westbury, NY). Bovine serum albumin (BSA) was purchased from Millipore-Sigma (St. Louis, MO). HEPES buffered saline-EDTA polysorbate (HBS-EP) buffer was purchased from GE Healthcare (Pittsburgh, PA). Tween20 was purchased from Bio-Rad (Hercules, CA). All other chemicals were purchased from Sigma Aldrich (St. Louis, MO) unless specified.

\section{Nonclinical Sample Information, Ligand Binding Drug Concentration, and ADA Assays}

REGN-P serum samples were obtained from a multipledose toxicology study in cynomolgus monkeys. Animals received $1 \mathrm{mg} / \mathrm{kg}$ REGN-P weekly (QW) via intravenous (IV) injection. Serum samples were collected at pre-dose, post-dose at $5 \mathrm{~min}, 1$ day, and 3 days within the first dosing interval, post-dose at 7, 15, 29, 43, 57, 71, 85, 106, 120, 134, 148 , and 162 days. REGN-N serum samples were obtained from a single-dose pharmacokinetic (PK) study in cynomolgus monkeys. Animals received a single IV injection of $1 \mathrm{mg} / \mathrm{kg}, 5 \mathrm{mg} / \mathrm{kg}$, or $15 \mathrm{mg} / \mathrm{kg}$ REGN-N. Serum samples were collected at pre-dose and post-dose at $5 \mathrm{~min}, 1,4,8,12$, $24,48,72,96,120,144,168,216,240,264,288,312,336,384$, 432, 480, 528, 576, 624, 672, 720, 768, 816, 864, 912, 960, 1008, $1056,1104,1152,1200,1272$, and $1344 \mathrm{~h}$ following the end of infusion. Serum samples were stored at $-80{ }^{\circ} \mathrm{C}$ prior to analysis.

REGN-P and REGN-N serum concentrations in serum were measured using a target capture enzyme-linked immunosorbent assay (ELISA). Microtiter plates were coated with the respective drug targets and the therapeutic captured on the plate was detected using a biotinylated mouse anti-human IgG4 monoclonal antibody, followed by Neutravidin conjugated with horseradish peroxidase. A luminol-based substrate, specific for peroxidase, was added to generate a signal intensity that is proportional to REGN-P and REGN-N concentration.

Anti-REGN-P antibodies in monkey serum samples were detected using an ECL-based bridging immunoassay. Serum samples were diluted 1:10 with $300 \mathrm{mM}$ acetic acid to dissociate antibody-drug complexes and were then diluted 1:3 with a $150-\mathrm{mM}$ Tris solution containing biotinylated and ruthenium-labeled REGN-P. ADAs in the serum samples form immune complexes with the labeled mAbs. The samples were added to a streptavidin-coated MSD microplate (Meso Scale Diagnostics, Rockville, MD). The bound complexes were detected by an electrochemiluminescent signal using an MSD plate reader (Meso Scale Discovery, Rockville, MD).

\section{Selection of Quantifying Peptides and Confirming Peptides for LC-MS/MS Assay}

Due to high homology shared between monkey and human IgGs, sequence alignment was performed to determine the unique peptides of each cynomolgus monkey $\mathrm{IgG}$ subclass $(23,24)$. Tryptic peptides derived from purified cynomolgus monkey $\operatorname{IgG}, \operatorname{IgA}$, and $\operatorname{IgM}$ were analyzed by LC-MS/MS using data-dependent acquisition (DDA) method on an Acquity I-Class UPLC system (Waters, Milford, MA) coupled to a Q Exactive Plus mass spectrometer (Thermo Fisher Scientific, San Jose, CA). Unique peptides for each isotype were selected to generate an MRM method. The most abundant $\mathrm{b}$ and $\mathrm{y}$ ions after the collision for each unique peptide were used to develop the MRM transitions, with the collision energy optimized for each transition. Tryptic peptides from purified monkey isotypes were analyzed using the MRM method on a 6495 triple quadrupole mass spectrometer (Agilent Technologies, Santa Clara, CA). The unique peptide for each ADA isotype with the strongest signal was chosen as the quantifying peptide (Table I) and any additional peptides were used as confirming peptides to certify the presence of ADA isotypes (Table S1). The top 2 transitions for each quantifying peptide were used for quantitation.

\section{Preparation of ADA-PC Standards}

ADA-PC standards in pooled monkey serum were prepared by performing a 3-fold serial dilution using pooled naïve monkey serum; final concentrations were $10 \mu \mathrm{g} / \mathrm{mL}$, $3.3 \mu \mathrm{g} / \mathrm{mL}, 1.1 \mu \mathrm{g} / \mathrm{mL}, 370 \mathrm{ng} / \mathrm{mL}, 123 \mathrm{ng} / \mathrm{mL}, 41 \mathrm{ng} / \mathrm{mL}$, $14 \mathrm{ng} / \mathrm{mL}$, and $1.5 \mathrm{ng} / \mathrm{mL}$. The standards were incubated at room temperature for $30 \mathrm{~min}$ before immunocapture.

\section{Preparation of Samples for Assay Drug Tolerance Assessment}

The drug tolerance samples were prepared by diluting REGN-P or REGN-N into pooled naïve monkey serum containing $500 \mathrm{ng} / \mathrm{mL}$ ADA-PC in a 2-fold serial dilution, resulting in a final concentration of drug ranging from $0.38 \mathrm{ng} /$ $\mathrm{mL}$ to $10 \mu \mathrm{g} / \mathrm{mL}$.

\section{Immunocapture with Biotinylated Drug}

Equal volumes of the individual monkey serum sample and $600 \mathrm{mM}$ acetic acid were combined and incubated at room temperature for $1 \mathrm{~h}$. The acidified serum samples were then diluted with HBS-EP buffer $+0.2 \%$ Tween-20. Biotinylated REGN-P or REGN-N (produced in-house, $2 \mu \mathrm{g} / \mathrm{sample}$ ) was added to each serum sample (final volume of $500 \mu \mathrm{L}$ ) and incubated for $1 \mathrm{~h}$ at room temperature to form a complex with ADA-PC or ADAs. Ten (10) microliter of Dynabeads MyOne ${ }^{\mathrm{TM}}$ Streptavidin T1 magnetic beads (Thermo Fisher Scientific, Waltham, MA) were incubated with 3\% BSA in HBS-EP $+0.2 \%$ Tween-20 buffer for 30 min before being added to the serum samples and subsequently incubated at room temperature for $15 \mathrm{~min}$. The samples were then washed twice with $3 \%$ BSA in HBS-EP $+0.2 \%$ Tween- 20 buffer followed by 2 washes with HBS-EP $+0.2 \%$ Tween- 20 buffer and elution with $0.1 \%$ formic acid (FA) and $50 \%$ acetonitrile.

\section{Immunocapture with Cross-linked Drug}

REGN-P and REGN-N were cross-linked to streptavidin beads using Dynabeads ${ }^{\mathrm{TM}}$ Antibody Coupling Kit (Thermo Fisher Scientific, Waltham, MA) according to the manufacturer's protocol to a final concentration of $50 \mathrm{ng} / \mathrm{mL}$. The beads with cross-linked MABs $(40 \mu \mathrm{L})$ for each sample were pre-blocked by incubating with $3 \%$ BSA in HBS-EP $+0.2 \%$ Tween-20 buffer for $30 \mathrm{~min}$. Acidified serum samples were 
Table I. List of Cynomolgus Monkey ADA Isotypes, Quantifying Peptides, MRM Transitions and Optimized Collision Energies Used in the Immunocapture-LC-MS Assay

\begin{tabular}{|c|c|c|c|c|c|}
\hline Isotype & Peptide & Precursor & Product ion & Collision energy & Note \\
\hline IgG1 & GPSVFPLAPSSR & 607.8300 & $\begin{array}{l}874.4781 \\
727.4097\end{array}$ & $\begin{array}{l}16.8 \\
16.8\end{array}$ & Unique for IgG1; quantifying peptide \\
\hline IgG2 & GPSVFPLASCSR & 639.3190 & $\begin{array}{l}937.4560 \\
790.3876\end{array}$ & $\begin{array}{l}23.8 \\
23.8\end{array}$ & Unique for $\operatorname{IgG} 2$; quantifying peptide \\
\hline IgG3 & GPSVFPLVSCSR & 653.3346 & $\begin{array}{l}965.4873 \\
818.4189\end{array}$ & $\begin{array}{l}27.3 \\
27.3\end{array}$ & Unique for IgG3; quantifying peptide \\
\hline IgG4 & GPSVFPLASSSR & 602.8197 & $\begin{array}{l}864.4574 \\
717.3890\end{array}$ & $\begin{array}{l}22.7 \\
22.7\end{array}$ & Unique for IgG4; quantifying peptide \\
\hline $\operatorname{IgM}$ & QIEVSWLR & 515.7876 & $\begin{array}{l}789.4254 \\
561.3144\end{array}$ & $\begin{array}{l}17.0 \\
17.0\end{array}$ & Unique for IgM; quantifying peptide \\
\hline $\operatorname{Ig} \mathrm{A}$ & DPSGATFTWTPSSGK & 769.8597 & $\begin{array}{l}863.4258 \\
475.2511\end{array}$ & $\begin{array}{l}30.9 \\
30.9\end{array}$ & Unique for IgA; quantifying peptide \\
\hline
\end{tabular}

diluted using HBS-EP buffer with $0.2 \%$ Tween-20 (total volume of $500 \mu \mathrm{L}$ ) and added to the pre-blocked cross-linked $\mathrm{MABs} /$ bead solution. The mixture was incubated at room temperature for $1 \mathrm{~h}$ to form MAB-ADA or MAB-ADA-PC complexes. The complexes on the magnetic bead were washed twice with $3 \%$ BSA in HBS-EP $+0.2 \%$ Tween-20 buffer followed by two washes of HBS-EP $+0.2 \%$ Tween-20 buffer and elution with $0.1 \%$ formic acid (FA) and $50 \%$ acetonitrile.

\section{Trypsin Digestion}

The eluted ADA-PC or ADAs were dried down in a SpeedVac concentrator and then resuspended in $20 \mu \mathrm{L}$ of $100 \mathrm{mM}$ Tris- $\mathrm{HCl} \mathrm{pH} 7.4$ containing $8 \mathrm{M}$ urea and $10 \mathrm{mM}$ of tris(2-carboxyethyl) phosphine (TCEP). The samples were incubated at $37^{\circ} \mathrm{C}$ for $30 \mathrm{~min}$. Trypsin (Promega, Sunnyvale, CA) was used at an enzyme: substrate ratio of $1: 10$, and $2.5 \mathrm{mM}$ iodoacetamide was added for alkylation. The samples were incubated in the dark at $37{ }^{\circ} \mathrm{C}$ for $3 \mathrm{~h}$. The digestion was terminated by the addition of $1 \mu \mathrm{L}$ of $20 \%$ formic acid. Twenty (20) microliter aliquots of the subsequent peptides were used for analysis.

\section{Mass Spectrometry and Data Analysis}

LC-MS/MS analyses were performed using an Acquity UPLC CSH C18 $1.7 \mu \mathrm{m}, 2.1 \mathrm{~mm} \times 50 \mathrm{~mm}$ column (Waters, Milford, MA) at $40{ }^{\circ} \mathrm{C}$ on a 1290 Infinity II LC System (Agilent, Santa Clara, CA) coupled with an Agilent 6495 triple quadrupole mass spectrometer. Mobile phase A consisted of $0.1 \%$ formic acid (FA) in water, and mobile phase B was composed of $0.1 \%$ FA in acetonitrile. Tryptic peptides were separated at a flow rate of $0.4 \mathrm{~mL} / \mathrm{min}$ with a linear gradient from $5 \%$ mobile phase B to $25 \%$ mobile phase B over 8 min. The column was then washed at $90 \%$ mobile phase $\mathrm{B}$ for $1 \mathrm{~min}$ and re-equilibrated to $5 \%$ mobile phase B for $2 \mathrm{~min}$ with a total analysis time of $15 \mathrm{~min}$. The MRM mass spectrometer settings were as follows: polarity was positive mode, MS1 and MS2 resolution was wide unit, dwell time was $50 \mathrm{~ms}$, and 12 quantifying and 16 confirming MRM transitions were monitored at various collision energy as described in
Table I and Table S1, respectively. The MRM-MS data was analyzed using Skyline (v4.1, MacCoss Lab, the University of Washington in Seattle, WA). Total peak areas from two fragment ions for each quantifying peptide were used for quantitation.

\section{RESULTS}

\section{Immunocapture LC-MRM-MS Workflow for ADA Isotyping and Semi-Quantitation}

Since singleplex bridging immunogenicity assays are not capable of determining ADA isotypes, we wanted to use the LC-MS platform to simultaneously detect, isotype, and quantitate ADA responses in monkeys administered different mAb therapeutics. MRM, also known as selected reaction monitoring (SRM), is a powerful and reliable technology to quantify low abundant proteins in complex matrices $(25,26)$. Here, we used human mAb drugs as the capture reagents to isolate ADA from monkey serum samples. The bound drugADA complexes were washed to eliminate contamination from serum proteins, specifically endogenous immunoglobulins. The isolated ADAs were then digested with trypsin to generate proteolytic peptides and analyzed in the triple quadrupole mass spectrometer using an MRM method (Fig. 1). A calibration curve generated by the ADA-PC was used to quantitate the amount of each isotype (Fig. 4).

\section{Selection of Surrogate Peptides}

In this MRM method, predefined transitions (precursor and fragment ion pair) were used to monitor and quantify specific proteins. A unique surrogate peptide corresponding to a specific immunoglobulin isotype was selected to generate this MS method. Similar to humans, monkeys have five heavy chain isotypes: $\operatorname{IgG}, \operatorname{IgA}, \operatorname{IgM}$, IgE, and $\operatorname{IgD}$; and two light chain isotypes: kappa and lambda. Monkeys also have four $\mathrm{IgG}$ subclasses, IgG1, IgG2, IgG3, and IgG4. Since $\mathrm{IgD}$ is mainly present in plasma membranes and rarely found in serum, it was not included in this assay. In addition, IgE contributes to less than $<0.003 \%$ of total immunoglobulin in serum and was therefore excluded from this assay as well. 


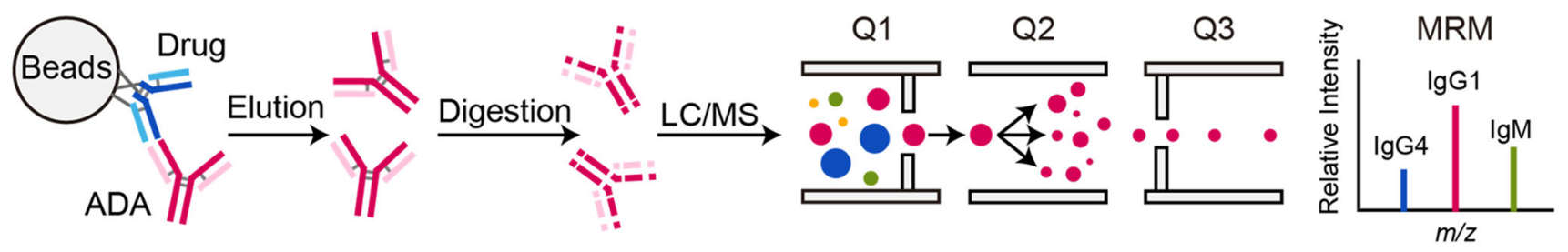

Fig. 1. Experimental workflow. Beads with cross-linked mAb drugs are added to the serum samples to form a complex with ADAs. Bound ADAs are then washed, eluted, digested, and analyzed using an LC-MRM-MS method for isotyping and quantitation

Several criteria were used for the selection of surrogate peptides. First, the surrogate peptides of each isotype/subclass must be within the constant regions of the antibody. Second, the peptides must be unique to each isotype/subclass and unique to monkey species. Third, they should generate strong MS signals. And finally, the peptides should not bear certain post-translational modifications, chemically induced modifications, and cleavage sites. For example, peptides with glycosylation sites or those containing methionine that is prone to oxidation were avoided. In addition, peptides with two neighboring basic amino acids (RR or KK), lysine-proline (KP), or arginine-proline (RP) were avoided to minimize inconsistent trypsin digestion. Peptides that met these criteria were measured by MS. The peptide with the most intense signal for precursor and fragment ions was chosen as the quantifying peptide for each isotype. An example of the MRM transition development was shown in Fig. S1. One or two additional peptides, other than the quantifying peptide if available, were used as confirming peptides to further certify the presence of ADA isotypes. The complete list of surrogate peptides and transitions for quantifying and confirming are listed in Table I and Table S1, respectively.

Cross-linking Drug to Streptavidin Magnetic Beads Significantly Increases ADA Immunocapture Recovery Rate Compared to Binding Biotinylated Drug to Streptavidin Magnetic Beads

Since the serum is a highly complex matrix, immunocapture enrichment was required to achieve the sensitivity necessary to distinguish potentially low abundant ADAs from other endogenous immunoglobulins using a standard LC-MS method. We compared two immunocapture approaches: biotinylating $\mathrm{mAb}$ drug with streptavidin-coated magnetic beads and directly cross-linking mAb drug to magnetic beads. A monkey anti-human kappa light chain IgG1 monoclonal antibody, ADA-PC, was used as a positive control to evaluate the recovery rate. To accurately assess the immunocapture recovery rate, mouse serum, instead of monkey serum, was used as the matrix to eliminate the interference from nonspecific binding of endogenous monkey immunoglobulins to ADA-PC.

First, we tested the biotinylated drug's ability to capture ADAs in sera using a method that has also been reported by others (19). Like ECL or ELISA-based ligand binding assays, immobilized streptavidin beads were used to isolate the drugADA complexes. Two biotinylated drugs, REGN-P and REGN-N, were used to immunocapture ADA-PC from serum samples. Compared to the input, which represents the amount of ADA-PC that was not subjected to immunocapture procedures but went through the same digestion and LC-MS procedures as samples, about $50 \%$ of the ADA-PC was detected after immunocapture for both REGN-P and REGN-N (Fig. 2). Second, we cross-linked each drug directly to magnetic beads before incubating them with serum samples containing ADAPC. The immunocapture recovery rate was improved significantly, with REGN-P achieving 89\% and REGN-N achieving $98 \%$ compared to the input (Fig. 2). Our results suggested that the direct cross-linking approach significantly improved the immunocapture recovery rate of ADA from mouse serum. Thus, the cross-linking approach was used in the final workflow.

Using Fab-Only Drug as the Capture Reagent Significantly Reduced Nonspecific Binding to the Fc Region of IgG4 Drugs

Human IgG4 antibodies are known to interact with other immunoglobulins via their Fc domain and can generate substantial background noise in immunoassays (27-29). Monkey IgG4 in serum samples may also bind via its Fc to the IgG4 drug cross-linked to magnetic beads in the immunocapture step, generating background in the assay. To test this, we compared full-length REGN-P and REGN-N to their corresponding Fab versions for the ADA immunocapture. Notably, high IgG4 background noise levels were detected when using full-length mAbs in the immunocapture step. This was substantially reduced when using Fab fragments to isolate ADAs (Fig. 3a). Two IgG1 drugs, REGN-6P and REGN-2P, were also tested with the

Immunocapture Recovery Rate

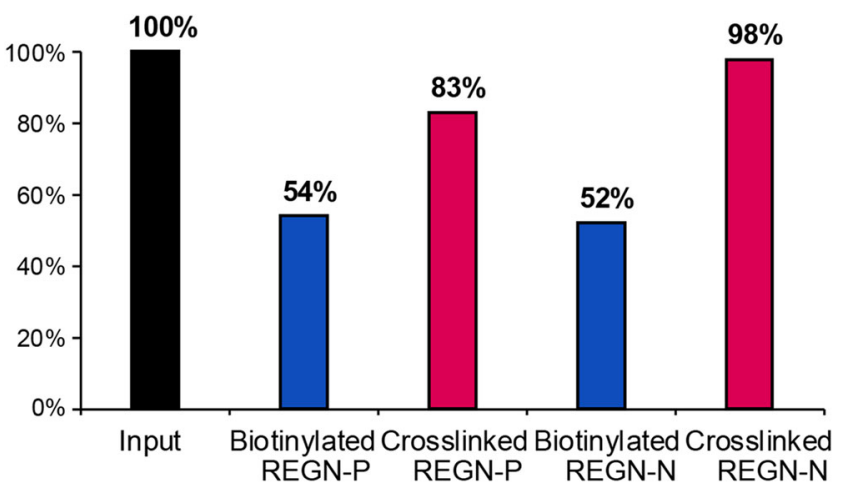

Fig. 2. Cross-linking drug to magnetic beads significantly increases ADA immunocapture recovery rate. Two IgG4 mAb drugs REGN-P and REGN-N were used as capture reagents. Biotinylated drugs coupled to streptavidin-coated beads and drugs directly cross-linked to beads were used to immunocapture ADAs in mouse serum. The input is the same amount of ADA-PC that did not go through immunocapture procedures but went through the same digestion procedures as the samples 


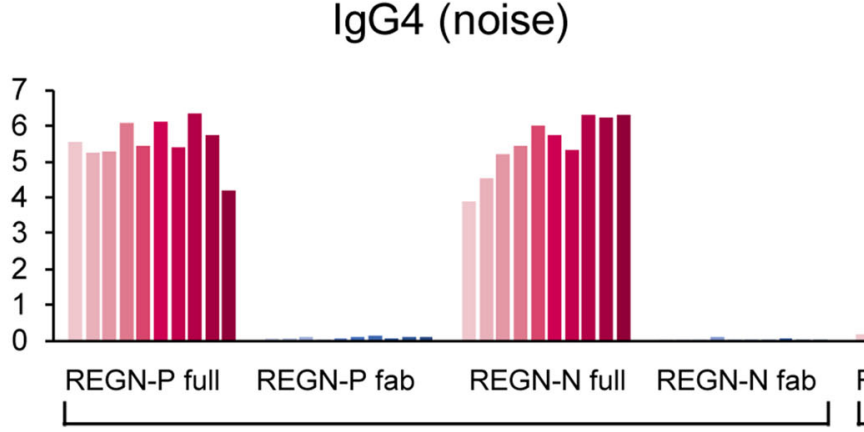

$\lg G 4$ drugs

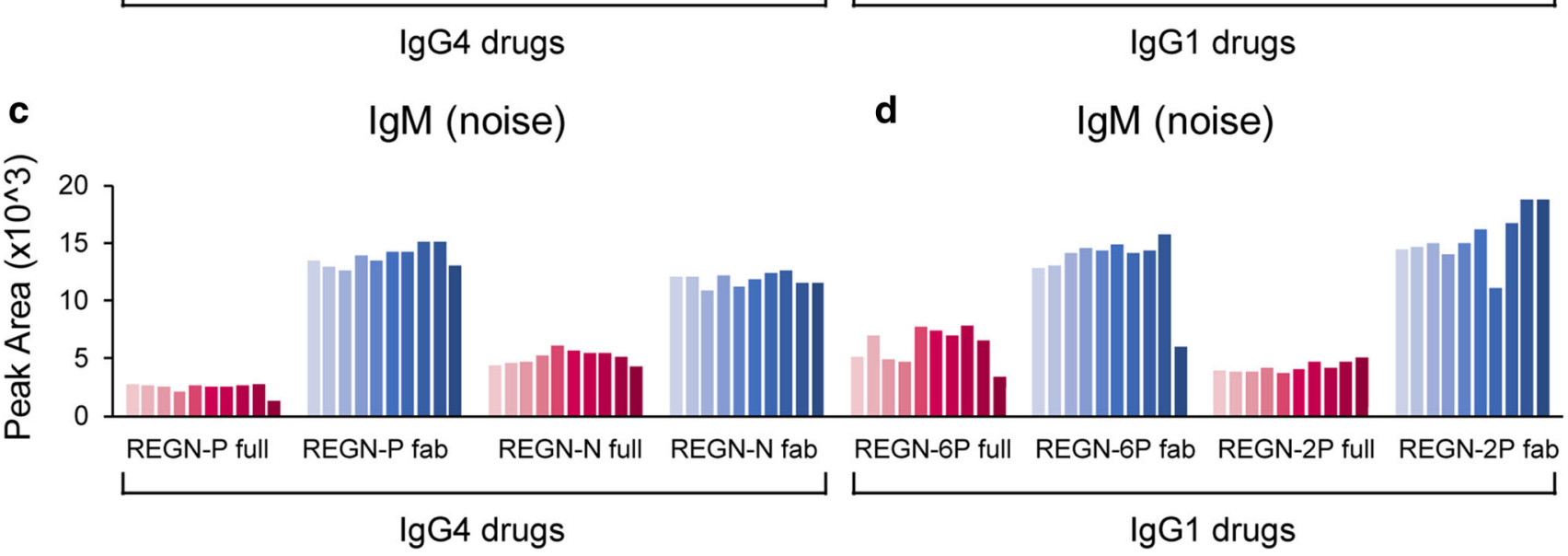

b

b

$\operatorname{lgG} 4$ (noise)

ADA concentration $(\mathrm{ng} / \mathrm{mL})$

Fig. 3. Fab-only drugs eliminate nonspecific interactions with serum components to the Fc region of IgG4 drugs. (a, b) Two IgG4 full-length $\mathrm{mAb}$ drugs and Fab-only drugs, and two IgG1 full-length mAb drugs and Fab-only drugs were used as capture reagents. In contrast to IgG4 drugs, IgG1 drugs do not have high IgG4 noise. IgG4 Fab-only drugs have significantly lower IgG4-mediated noise comparing to IgG4 full drugs. (c, d) For both IgG4 and IgG1 drugs, Fab-only drugs cause higher IgM-mediated background noise

full-length and Fab versions to determine if other isotypes could generate similar background noise. As expected, the two IgG1 drugs did not generate high IgG4 background noises (Fig. 3b).

In contrast, $\operatorname{IgM}$ matrix interference was observed for both IgG1 and IgG4 drugs (Fig. 3c \& d), when both fulllength $\mathrm{mAb}$ and $\mathrm{Fab}$ were used in the immunocapture step. Interestingly, there were greater levels of IgM background noise when the Fab was used for immunocapture. Because of the significant reduction of nonspecific interactions, Fab-only drugs cross-linked to magnetic beads were used as the immunocapture reagents in the final workflow.

\section{Determination of LLOQ and ULOQ of the Assay Using a Standard Calibration Curve}

Standard calibration curves used for ADA quantitation were developed for both REGN-P and REGN-N drugs. ADA-PC was serially diluted with pooled naïve monkey serum from $10 \mu \mathrm{g} / \mathrm{mL}$ to $1.5 \mathrm{ng} / \mathrm{mL}$, using a 3-fold dilution factor. The positive control samples were processed with the optimized immunocapture method procedure with crosslinked REGN-P Fab and cross-linked REGN-N Fab as the capture reagents. The isolated ADA controls were then analyzed by the 15 -min LC-MS method in triplicate. MRM transitions of GPSVFPLAPSSR peptide from IgG1 were used for quantitation (Table I). The peak area corresponding to each ADA-PC concentration was used to plot the calibration curve. The REGN-P calibration curve showed a linear range from $41 \mathrm{ng} / \mathrm{mL}$ to $10 \mu \mathrm{g} / \mathrm{mL}$ (Fig. 4a) and the REGN-N calibration curve showed a linear range from $14 \mathrm{ng} /$ $\mathrm{mL}$ to $10 \mu \mathrm{g} / \mathrm{mL}$ (Fig. 4b). For REGN-P, the limit of detection (LOD) was determined to be $1.5 \mathrm{ng} / \mathrm{mL}$ using a single-tonoise $(\mathrm{S} / \mathrm{N})$ ratio of 3 and the lower limit of quantitation (LLOQ) was determined to be $41 \mathrm{ng} / \mathrm{mL}$ using an $\mathrm{S} / \mathrm{N}$ ratio of 10 , as well as in the linear range of the calibration curve. For REGN-N, the limit of detection (LOD) was determined to be $1.5 \mathrm{ng} / \mathrm{mL}$ using a single-to-noise $(\mathrm{S} / \mathrm{N})$ ratio of 3 and the lower limit of quantitation (LLOQ) was determined to be $14 \mathrm{ng} / \mathrm{mL}$ using an $\mathrm{S} / \mathrm{N}$ ratio of 10 , as well as in the linear range of the calibration curve. The upper limit of quantitation (ULOQ) for both REGN-P and REGN-N were determined to be $10 \mu \mathrm{g} / \mathrm{mL}$ as the upper limit of the linear range of standard calibration curves.

\section{Determination of Drug Tolerance Limit}

The presence of therapeutic drugs in nonclinical monkey serum samples may compete with immunocapture reagents for binding to ADAs, also known as drug interference. To assess the assay tolerance to REGN-P and REGN-N drugs present in serum samples, pooled monkey serum samples containing $500 \mathrm{ng} / \mathrm{mL}$ of ADA-PC were tested in the presence of different concentrations of REGN-P or REGN$\mathrm{N}$ (ranging from $0.38 \mathrm{ng} / \mathrm{mL}$ to $10 \mu \mathrm{g} / \mathrm{mL}$ ) to determine what concentrations of drug impacts on ADA quantitation and ADA detection. 


\section{a REGN-P Calibration Curve}

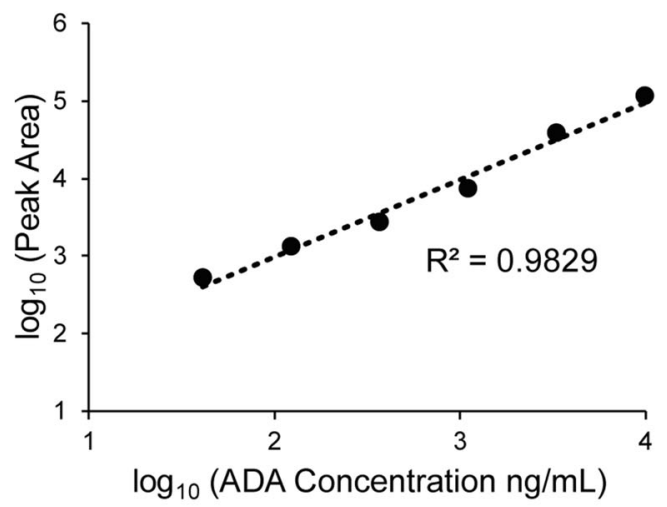

b REGN-N Calibration Curve

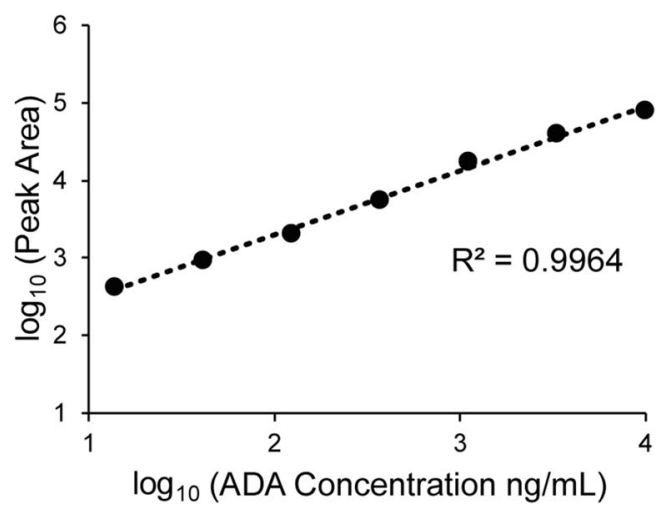

Fig. 4. ADA calibration curves of REGN-P and REGN-N. Calibration curve of REGN-P (a) and REGN-N (b). The $x$-axis represents the log scale of ADA concentration while the $y$-axis represents the $\log$ scale of peak area of IgG1 quantifying peptide, GPSVFPLAPSSR, quantified using the MRM method. The lowest point in (a) corresponds to $41 \mathrm{ng} / \mathrm{mL}$ of ADAs. The lowest point in (b) corresponds to $14 \mathrm{ng} / \mathrm{mL}$ of ADAs

The quantitation of ADA-PC was not impacted by REGN-P or REGN-N until the drug concentration reached $195 \mathrm{ng} / \mathrm{mL}$ (Fig. 5). When the drug concentration was higher than $195 \mathrm{ng} / \mathrm{mL}$, the concentration of ADA-PC detected in the assay started to decrease, indicating the competing effect of the drug in the sera for ADA binding.

Ligand binding ADA assays are non-quantitative and ADA concentrations in nonclinical studies are reported as either negative or positive with a titer (dilution) value which provides a relative quantitation of the antibody responses. In the drug tolerance experiments, ADA-PC can still be detected even at REGN-P or REGN-N concentration of $3.1 \mu \mathrm{g} / \mathrm{mL}$, with the ADA-PC signal 3-fold greater than assay background at this drug concentration.

\section{Application of Optimized Method to Nonclinical Monkey} Serum Samples for ADA Isotyping and Semi-Quantitation

To understand the ability of the LC-MS method to detect and isotype actual ADA responses, nonclinical samples from monkeys administered REGN-P or REGN$\mathrm{N}$ were assessed in the LC-MS assay. In one study, monkeys were administered REGN-P intravenously every week for 26 weeks. Two animals that had positive ADA responses detected by an ECL-based ligand binding assay were chosen to be evaluated by our LC-MS method. For monkey 1 , a strong positive response was detected in the LBA assay, whereas a weakly positive response was detected in monkey 2 (Fig. 6e). In terms of the drug concentration-time profile for monkey 1 , there was an accelerated decrease in serum concentrations which likely reflects the ADA impact on the circulating drug levels. (Fig. 6a). For this monkey with accelerated drug clearance, ADA isotypes $\mathrm{IgG} 1, \mathrm{IgG} 2$, and $\mathrm{IgG} 4$ were detected by the LC-MS method. IgG1 was the most abundant isotype (Fig. 6b). It was detected on study day 43 and generally increased throughout the study. IgG2 and IgG4 showed similar increases from day 43 to day 134. Notably, starting from day 57, the concentration of REGN-P in monkey 1 serum samples was below the assay drug tolerance limit of $195 \mathrm{ng} / \mathrm{mL}$ (Table II).

For monkey 2, relatively little ADAs were detected with the LC-MS method which is consistent with the low responses in the LBA assay and the lack of accelerated clearance observed in the concentration vs time profile. Nevertheless, as with monkey $1, \operatorname{IgG} 1$ was the most abundant isotype, followed by $\mathrm{IgG} 4$ and $\mathrm{IgG} 2$ (Fig. 6c). It should be noted that the REGN-P drug concentrations for monkey 2 were above the assay drug tolerance limit of $195 \mathrm{ng} / \mathrm{mL}$ for the majority of the analyzed time points, and therefore, the levels of ADAs in these time points may be underestimated. (Table II).

After successfully isotyping ADAs, the peak areas were summed for all isotypes to calculate the total ADA concentrations in the REGN-P-dosed monkey serum samples using the REGN-P-captured ADA-PC calibration curve (Fig. 6d). Pre-dose serum samples were used as individual negative controls. A signal-to-noise ratio was calculated by comparing sample peak areas to the negative control peak area. Serum samples from monkey 1 were strongly ADA-positive starting from day 43, with blood REGN-P levels at $1.94 \mu \mathrm{g} / \mathrm{mL}$ (Table II). Total ADA concentrations ranged from $340 \mathrm{ng} / \mathrm{mL}$ at day 43 to $5.75 \mu \mathrm{g} / \mathrm{mL}$ on day 162 . Monkey 2 serum samples were weakly ADA-positive starting on day 106, with blood REGN-P levels at $10 \mu \mathrm{g} / \mathrm{mL}$. The total ADA concentrations ranged from $350 \mathrm{ng} / \mathrm{mL}$ at day 106 to $720 \mathrm{ng} / \mathrm{mL}$ on day 162. Monkey 1 had a rapid increase in the levels of ADAs throughout the dosing period, corresponding to its steep decline in drug concentrations early on, whereas monkey 2 has very low levels of ADAs over the same time frame (Table II). Thus, the results obtained using the LC-MS based ADA quantitation method for both monkey 1 and monkey 2 were similar to those obtained with the ligand binding assay (Fig. 6e).

Nonclinical serum samples from animals administered REGN-N were also evaluated. Samples from three monkeys collected after a single-dose of REGN-N at doses of 1, 5 , or $15 \mathrm{mg} / \mathrm{kg}$ were analyzed. All three monkeys demonstrated a rapid decrease in REGN-N drug concentrations over time (Fig. 7a) and all animals exhibited robust positive responses in the LBA ADA assay (not shown). The LCMS ADA method also detected strong ADA responses in all 3 animals. The isotyping results suggested $\operatorname{IgG1}$ was the 


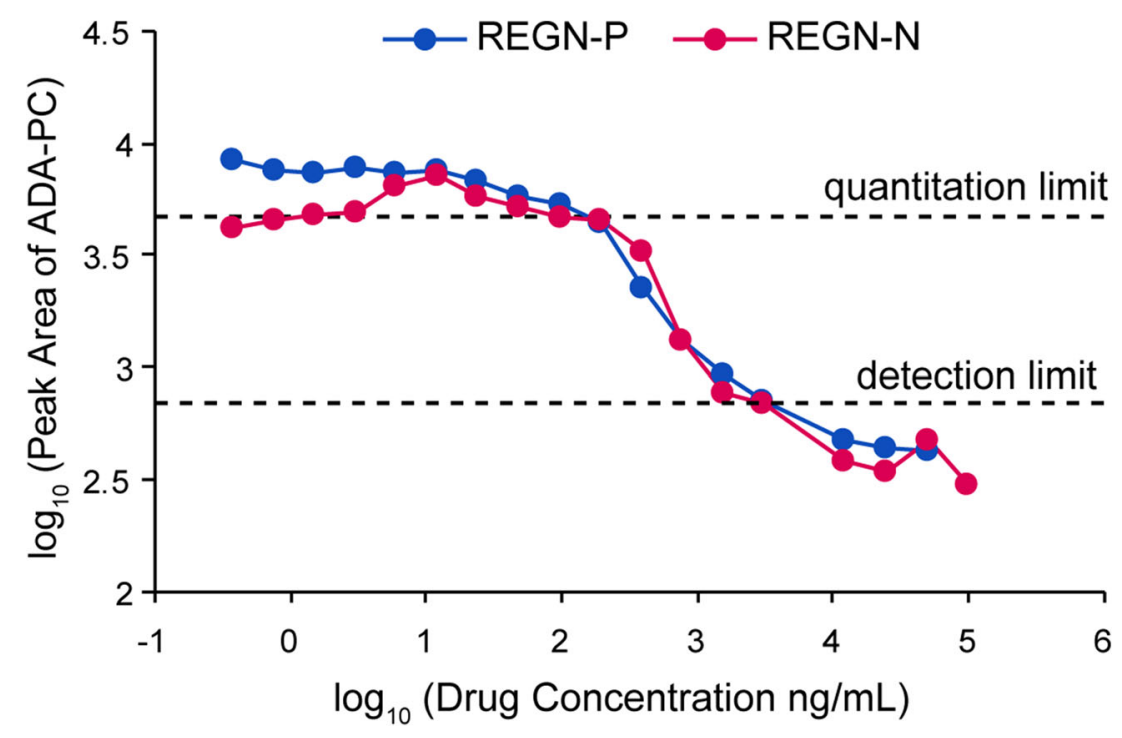

Fig. 5. Determination of drug tolerance limit. REGN-P or REGN-N was diluted into pooled naïve monkey serum containing $500 \mathrm{ng} / \mathrm{mL}$ of ADA-PC in a 2-fold serial dilution, resulting in a final concentration of drug ranging from $0.38 \mathrm{ng} / \mathrm{mL}$ to $10 \mu \mathrm{g} / \mathrm{mL}$. ADA levels were measured to assess the impact of the drug concentration on ADA quantitation and detection limits

most abundant ADA isotype for all these monkeys, with IgG2 presented in all three samples (Fig. 7b, c \& d). IgG4 was at a comparable level to $\mathrm{IgG} 2$ for monkey 2 but was significantly lower for monkey 3. All three monkeys were ADA-positive beginning on day 18, with ADA concentrations increasing over time (Fig. 7e). It should be noted that

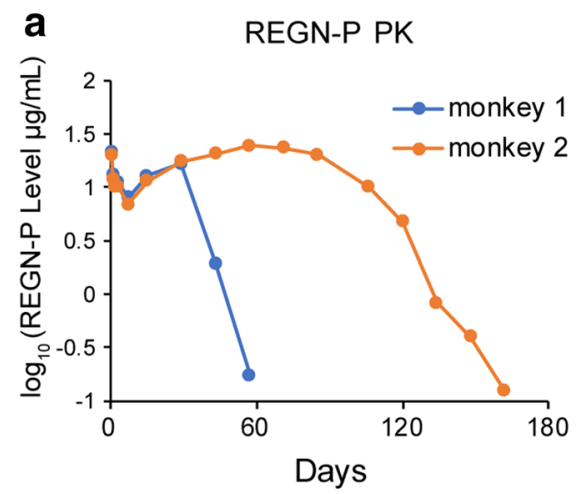

d

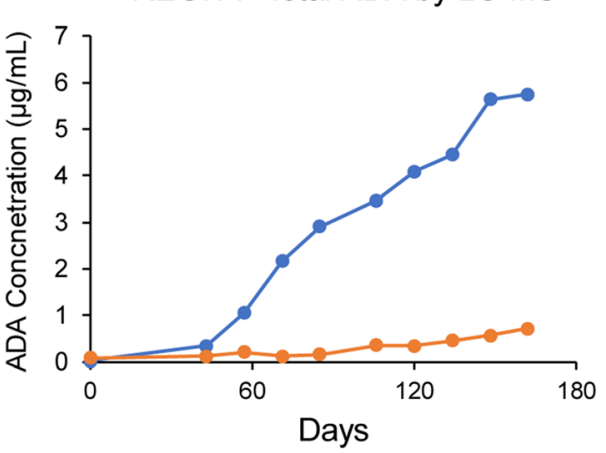

b ADA Isotyping: monkey 1
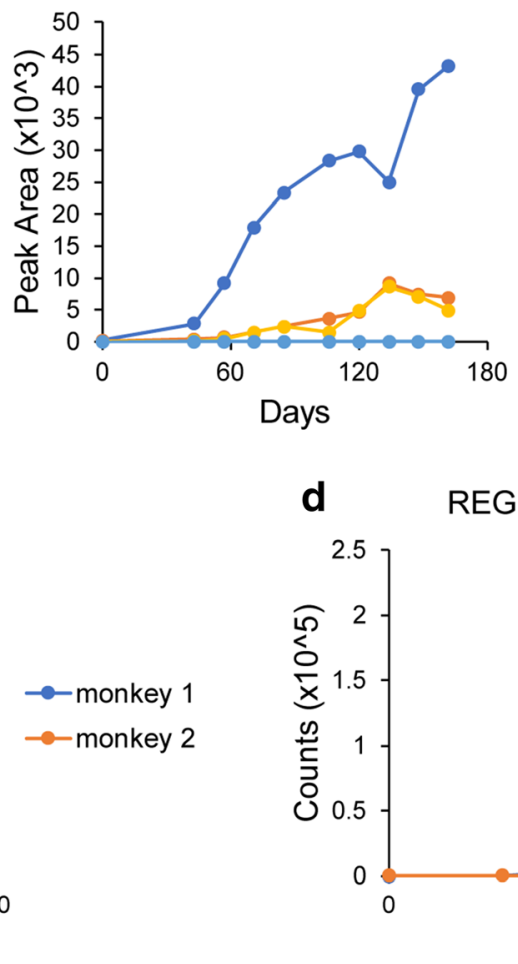

d REGN-P Total ADA by LBA

C ADA Isotyping: monkey 2
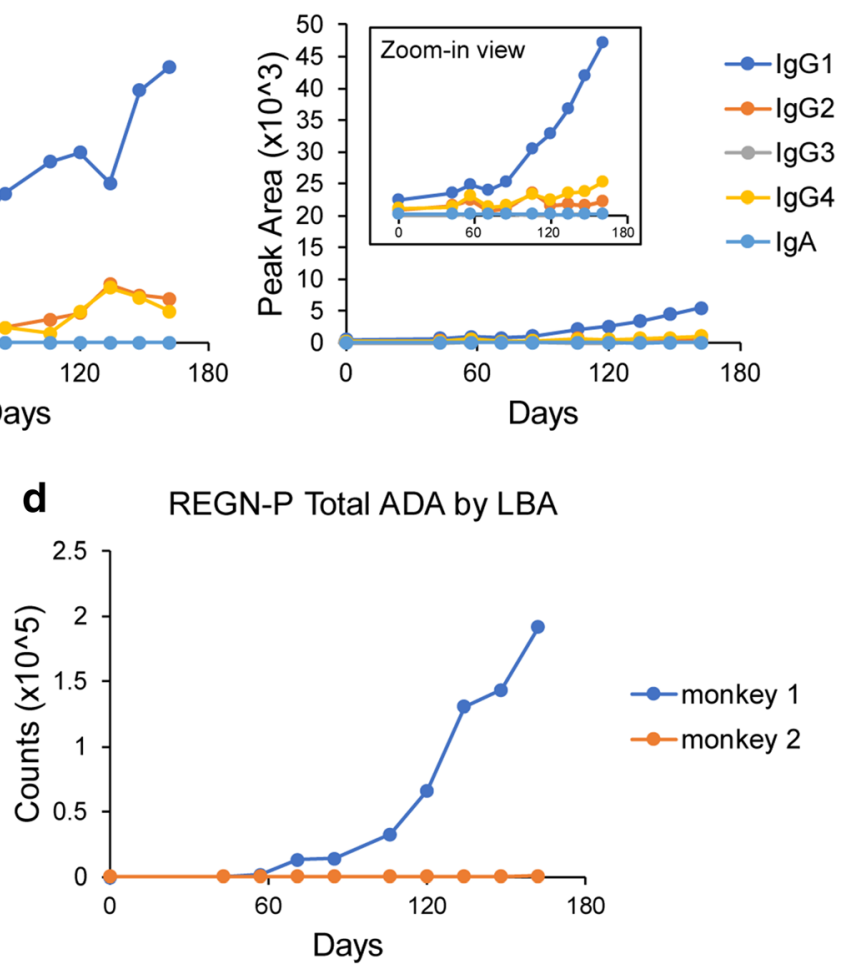

Fig. 6. REGN-P monkey serum sample ADA isotyping and quantitation. a PK results of two monkeys with weekly dose of $1 \mathrm{mg} / \mathrm{kg}$ REGN-P for 23 weeks. b ADA isotyping results of monkey 1 using the immunocapture LC-MS method. c ADA isotyping results of monkey 2 using the immunocapture LC-MS method. d Total ADAs were quantified using the calibration curve in Fig. 4a to convert the peak areas to ADA concentrations. e ADA assay signal (counts) using a ECL-based ligand binding assay 
Table II. Summary of REGN-P Drug Levels, Mass Spec Peak Areas of All ADA Isotypes, Converted ADA Concentrations, and Signal-toNoise Ratios

\begin{tabular}{|c|c|c|c|c|c|c|c|c|c|c|c|}
\hline Days & & 0 & 43 & 57 & 71 & 85 & 106 & 120 & 134 & 148 & 162 \\
\hline \multirow[t]{4}{*}{ Monkey 1} & Drug level $(\mu \mathrm{g} / \mathrm{mL})$ & BLQ & 1.94 & 0.174 & BLQ & BLQ & BLQ & BLQ & BLQ & BLQ & BLQ \\
\hline & Peak area & 278 & 3309 & 10,217 & 20,911 & 28,106 & 33,451 & 39,392 & 42,783 & 54,189 & 55,203 \\
\hline & ADA conc. $(\mu \mathrm{g} / \mathrm{mL})$ & 0.03 & 0.34 & 1.06 & 2.17 & 2.92 & 3.48 & 4.10 & 4.45 & 5.64 & 5.75 \\
\hline & Signal/noise & 1.0 & 11.9 & 36.8 & 75.2 & 101.1 & 120.3 & 141.7 & 153.9 & 194.9 & 198.6 \\
\hline \multirow[t]{4}{*}{ Monkey 2} & Drug level $(\mu \mathrm{g} / \mathrm{mL})$ & BLQ & 20.6 & 24.6 & 23.4 & 20.2 & 10 & 4.79 & 0.828 & 0.396 & 0.124 \\
\hline & Peak area & 829 & 1231 & 2002 & 1160 & 1521 & 3425 & 3371 & 4443 & 5507 & 6986 \\
\hline & ADA conc. $(\mu \mathrm{g} / \mathrm{mL})$ & 0.08 & 0.13 & 0.21 & 0.12 & 0.16 & 0.35 & 0.35 & 0.46 & 0.57 & 0.72 \\
\hline & Signal/noise & 1.0 & 1.5 & 2.4 & 1.4 & 1.8 & 4.1 & 4.1 & 5.4 & 6.6 & 8.4 \\
\hline
\end{tabular}

The signal/noise represents the ratio of peak area of a given sample to the pre-dose negative monkey serum sample

the REGN-N drug concentrations in the three monkeys were all below the drug tolerance limit $(3.1 \mu \mathrm{g} / \mathrm{mL})$ for ADA detection.

\section{DISCUSSION}

The optimization of the ADA immunocapture procedure with maximal recovery and minimal matrix interference is critical for the LC-MS ADA assay. Our results suggested direct cross-linking conjugation of drugs onto magnetic beads increased immunocapture recovery rate by twofold compared to the traditional conjugation of biotinylated drugs onto streptavidin magnetic beads (Fig. 2). This is most likely due to poorer labeling efficacy of biotin to drugs compared to cross-linking and due to higher conjugation density of crosslinking compared to bulkier biotin tags. The percentage of unlabeled drugs in both biotinylated REGN-P and REGN-N reagents was approximately 5\% (data not shown). The unbiotinylated drugs in the capture reagents would reduce the ADA capture recovery rate as the unbiotinylated drugs could not bind to streptavidin beads and thus were washed away along with any ADAs that bind to the unbiotinylated drugs. In contrast, uncross-linked drugs were washed away from the beads prior to the immunocapture step, resulting in a higher and more consistent ADA capture recovery rate.

Interestingly, when the full-length mAb drug was used as the capture reagent, there was a high level of $\mathrm{IgG} 4$ background noise. However, using the Fab fragment in the immunocapture step substantially reduced the background signal (Fig. 3). Human IgG4 can form Fc-Fc contacts which can cause interference in immunoassays, including ADA assays (27-29). The arginine at position 409 (EU numbering) is responsible for these Fc-Fc contacts, and the monkey IgG4 sequence is conserved with the human sequence at this residue (30). The results presented here indicate that Fc-Fc interactions also occur between monkey and human IgG4, but not with human IgG1.

Interference from endogenous monkey $\operatorname{IgM}$ was also observed in the immunocapture step. Our results suggest endogenous IgM binds to both IgG1 and IgG4 drugs and acid dissociation was unsuccessful in eliminating these interactions. When using the Fab-only drug as a capture reagent, interference levels increased twofold. Binding to the fulllength $\mathrm{mAb}$ in the immunocapture step may be due to endogenous anti-human IgM in monkey serum, or RF-type responses directed to the $\mathrm{Fc}$, as occur in humans (31). The increased binding to the Fab version of the drug may have been due to pre-existing anti-hinge antibodies that are not detected with the full-length mAb (32). We were not able to select surrogate peptides for IgE due to the lack of commercially available cynomolgus monkey $\operatorname{IgE}$.

The assay can be used to determine whether a sample is ADA-positive or negative by comparing assay response of a post-dose sample to that monkey's baseline response, which is sometimes used in nonclinical ADA assessment with LBA assays. For example, we can establish a threshold to determine ADA positivity if the ratio of the MS signals of post-dose samples to the MS signal of the pre-dose samples of the same animal is greater than 3. Using this threshold, REGN-P-dosed monkey 1 had a positive ADA response before day 43, while the first ADA-positive response for monkey 2 was observed at day 106 (Table II).

The assay can tolerate $195 \mathrm{ng} / \mathrm{mL}$ of REGN-P and REGN-N in monkey serum without affecting ADA quantitation and can tolerate $3.1 \mu \mathrm{g} / \mathrm{mL}$ of REGN-P and REGN-N in monkey serum without affecting ADA identification (Fig. 5). To further improve the drug tolerance and thus enable ADA quantitation at high drug concentrations, the sample could be pre-treated with anti-human antibodies to deplete the existing drugs. However, the capturing anti-human antibodies should not compete with endogenous ADAs for the same binding domain on the drug. Furthermore, a calibration curve was successfully used to quantify ADA levels with a dynamic range from $123 \mathrm{ng} / \mathrm{mL}$ (REGN-P) or $41 \mathrm{ng} / \mathrm{mL}$ (REGNN) to $10 \mu \mathrm{g} / \mathrm{mL}$. We speculate that stable heavy isotope-labeled ADA-PC can be spiked into serum samples to further improve the quantitation. Furthermore, since ADA-PC technically only represents IgG1 isotype, it will be ideal to generate a positive control cocktail composed of multiple ADA isotype positive standards against human $\mathrm{IgG}$ to achieve better quantitation.

\section{CONCLUSION}

An immunocapture LC-MS-based method was successfully employed to isotype and quantify ADAs in monkey serum samples that were previously determined to be ADApositive by a ligand binding assay. Consistent with previous work (9), our data suggest IgG1 is the predominant ADA isotype. $\mathrm{IgG} 2$ and $\mathrm{IgG} 4$ were also detected at lower levels, while $\operatorname{IgG} 3$ and $\operatorname{Ig}$ A levels were negligible. 


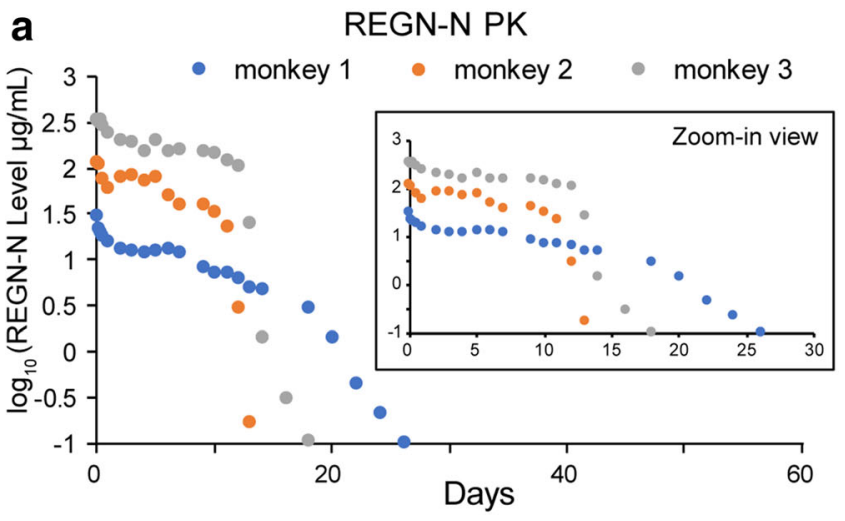

b

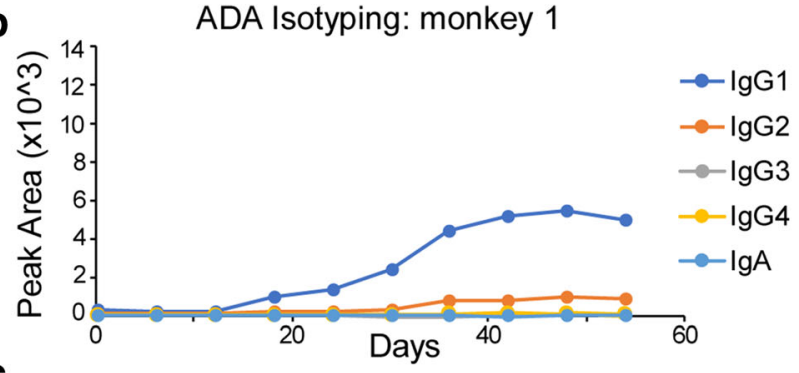

C

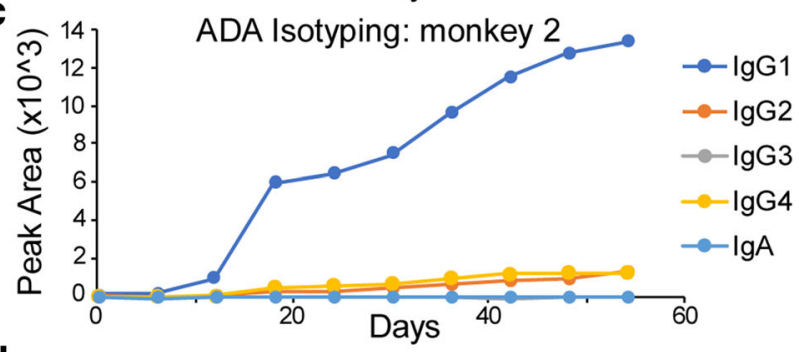

d

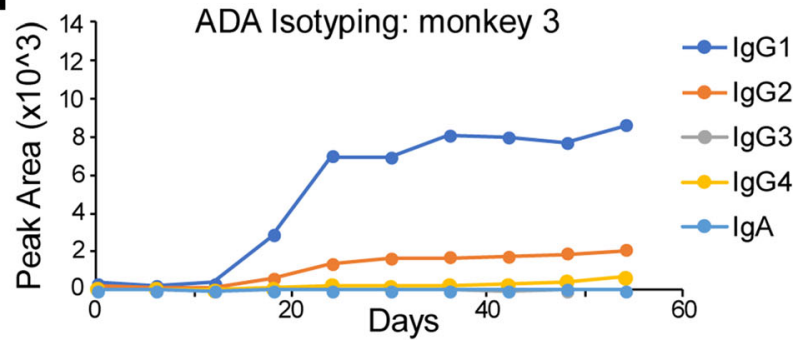

Fig. 7. REGN-N monkey serum sample ADA isotyping and quantitation. a PK results of monkeys 1,2 , and 3 with a single-dose of 1,5 , and $15 \mathrm{mg} / \mathrm{kg}$ REGN-N, respectively. ADA isotyping results using the immunocapture LC-MS method for monkey $1 \mathbf{b}$, monkey $2 \mathbf{c}$, and monkey 3 d. e ADAs were quantified using the calibration curve in Fig. $4 \mathrm{~b}$ to convert peak areas to concentrations

A monkey IgG1 mAb against human kappa light chain was used as a positive control for assay development and as a calibration standard. Several critical experimental conditions in the immunocapture step were optimized. Cross-linking conjugation of drugs to beads ensured maximal ADA recovery. A Fab-only drug was used as the capture reagent to eliminate the interference due to Fc interactions with IgG4 drugs. The multiplex LC-MRMMS method allows simultaneously to monitor 6 ADA isotypes using 17 MRM transitions with a short 15-min LC method.

$>$ In summary, this immunocapture LC-MS assay can provide specific ADA isotype and semi-quantitation information and can be used as a supplementary and orthogonal approach to traditional ADA assays for immunogenicity assessment.

\section{ACKNOWLEDGMENTS}

The authors would like to thank Laura Wedel and Ashley Roberts of Scientific Writing Group for their assistance in drafting and editing the manuscript.

\section{COMPLIANCE WITH ETHICAL STANDARDS}

All studies were conducted under protocols approved by the Institutional Animal Care and Use Committees of Charles River Laboratories and SNBL, Ltd; both are AAALAC accredited laboratories.
Open Access This article is licensed under a Creative Commons Attribution 4.0 International License, which permits use, sharing, adaptation, distribution and reproduction in any medium or format, as long as you give appropriate credit to the original author(s) and the source, provide a link to the Creative Commons licence, and indicate if changes were made. The images or other third party material in this article are included in the article's Creative Commons licence, unless indicated otherwise in a credit line to the material. If material is not included in the article's Creative Commons licence and your intended use is not permitted by statutory regulation or exceeds the permitted use, you will need to obtain permission directly from the copyright holder. To view a copy of this licence, visit http://creativecommons.org/licenses/by/4.0/.

\section{SUPPLEMENTARY INFORMATION}

The online version contains supplementary material available at https://doi.org/10.1208/s12248-020-00538-w.

\section{REFERENCES}

1. De Groot AS, Scott DW. Immunogenicity of protein therapeutics. Trends Immunol. 2007;28(11):482-90. https://doi.org/ 10.1016/j.it.2007.07.011. 
2. Krishna M, Nadler SG. Immunogenicity to biotherapeutics - the role of anti-drug immune complexes. Front Immunol. 2016;7:21. https://doi.org/10.3389/fimmu.2016.00021.

3. Baker MP, Reynolds HM, Lumicisi B, Bryson CJ. Immunogenicity of protein therapeutics: the key causes, consequences and challenges. Self Nonself. 2010;1(4):314-22. https://doi.org/ 10.4161/self.1.4.13904.

4. Wang YM, Wang J, Hon YY, Zhou L, Fang L, Ahn HY. Evaluating and reporting the immunogenicity impacts for biological products-a clinical pharmacology perspective. AAPS J. 2016;18(2):395-403. https://doi.org/10.1208/s12248-015-9857-y.

5. Singh SK. Impact of product-related factors on immunogenicity of biotherapeutics. J Pharm Sci. 2011;100(2):354-87. https:// doi.org/10.1002/jps.22276.

6. Kuriakose A, Chirmule N, Nair P. Immunogenicity of biotherapeutics: causes and association with posttranslational modifications. J Immunol Res. 2016;2016:1298473-18. https:// doi.org/10.1155/2016/1298473.

7. Wadhwa M, Knezevic I, Kang HN, Thorpe R. Immunogenicity assessment of biotherapeutic products: an overview of assays and their utility. Biologicals. 2015;43(5):298-306. https://doi.org/ 10.1016/j.biologicals.2015.06.004.

8. Swanson SJ, Bussiere J. Immunogenicity assessment in nonclinical studies. Curr Opin Microbiol. 2012;15(3):337-47. https:// doi.org/10.1016/j.mib.2012.05.015.

9. Vidarsson G, Dekkers G, Rispens T. IgG subclasses and allotypes: from structure to effector functions. Front Immunol. 2014;5:520. https://doi.org/10.3389/fimmu.2014.00520.

10. Monte-Wicher V, Wicher K, Arbesman CE. Comparative studies of monkey and human immunoglobulins. Immunochemistry. 1970;7(10):839-49. https://doi.org/10.1016/00192791(70)90060-1.

11. FDA. Guidance for industry: immunogenicity testing of therapeutic protein products developing and validating assays for anti-drug antibody detection. 2019.

12. Shibata H, Nishimura K, Miyama C, Tada M, Suzuki T, Saito Y, et al. Comparison of different immunoassay methods to detect human anti-drug antibody using the WHO erythropoietin antibody reference panel for analytes. J Immunol Methods. 2018;452:73-7. https://doi.org/10.1016/j.jim.2017.09.009.

13. $\mathrm{Xu} \mathrm{X}$, Qiu H, Li N. LC-MS multi-attribute method for characterization of biologics. J Appl Bioanalysis. 2017;3(2):215. https://doi.org/10.17145/jab.17.003.

14. $\mathrm{Xu} \mathrm{X}$. In vivo characterization of therapeutic monoclonal antibodies. J Appl Bioanalysis. 2016;2(1):6.

15. Xu X, Huang Y, Pan H, Molden R, Qiu H, Daly TJ, et al. Quantitation and modeling of post-translational modifications in a therapeutic monoclonal antibody from single- and multipledose monkey pharmacokinetic studies using mass spectrometry. PLoS One. 2019;14(10):e0223899. https://doi.org/10.1371/ journal.pone.0223899.

16. Chen L. Current status of anti-drug antibody analysis using immunocapture-liquid chromatography/mass spectrometry. J Appl Bioanalysis. 2018;4(3):15.

17. Neubert H, Grace C, Rumpel K, James I. Assessing immunogenicity in the presence of excess protein therapeutic using immunoprecipitation and quantitative mass spectrometry. Anal Chem. 2008;80(18):6907-14. https://doi.org/10.1021/ac8005439.

18. Jiang H, Xu WF, Titsch CA, Furlong MT, Dodge R, Voronin K, et al. Innovative use of LC-MS/MS for simultaneous quantitation of neutralizing antibody, residual drug, and human immunoglobulin $G$ in immunogenicity assay development. Anal Chem. 2014;86(5):2673-80. https://doi.org/10.1021/ac5001465.

19. Chen LZ, Roos D, Philip E. Development of immunocaptureLC/MS assay for simultaneous ADA isotyping and semiquantitation. J Immunol Res. 2016;2016:7682472-14. https://doi.org/10.1155/2016/7682472.

20. Roos D, Chen LZ, Vesapogu R, Kane C, Duggan J, Norris S. Detection of cynomolgus monkey anti-protein XYZ antibody using immunocapture-LC/MS. J Appl Bioanalysis. 2016;2(4):117-28. https://doi.org/10.17145/jab.16.016.

21. Chen LZ, Roos D, Philip E, Pagels S. Sequential immunoaffinity-LC/MS assay for quantitation of a therapeutic protein in monkey plasma. J Appl Bioanalysis. 2017;3(4):127-38.

22. Kobayashi K, Tahara H, Kagawa Y. A method combining blue native polyacrylamide gel electrophoresis with liquid chromatography tandem-mass spectrometry to detect circulating immune complexes between therapeutic monoclonal antibodies and anti-drug antibodies in animals. J Pharm Biomed Anal. 2020;186:113329. https://doi.org/10.1016/j.jpba.2020.113329.

23. Jacobsen FW, Padaki R, Morris AE, Aldrich TL, Armitage RJ, Allen MJ, et al. Molecular and functional characterization of cynomolgus monkey IgG subclasses. J Immunol. 2011;186(1):341-9. https://doi.org/10.4049/jimmunol.1001685.

24. Nguyen DC, Sanghvi R, Scinicariello F, Pulit-Penaloza J, Hill N, Attanasio R. Cynomolgus and pigtail macaque IgG subclasses: characterization of IGHG genes and computational analysis of $\mathrm{IgG/fc}$ receptor binding affinity. Immunogenetics. 2014;66(6):361-77. https://doi.org/10.1007/s00251-014-0775-4.

25. Lange V, Picotti P, Domon B, Aebersold R. Selected reaction monitoring for quantitative proteomics: a tutorial. Mol Syst Biol. 2008;4:222. https://doi.org/10.1038/msb.2008.61.

26. de Hoffmann E. Tandem mass spectrometry: a primer. J Mass Spectrom. 1996;31(2):129-37. https://doi.org/10.1002/ (SICI)1096-9888(199602)31:2<129::AID-JMS305>3.0.CO;2-T.

27. Aalberse RC, Stapel SO, Schuurman J, Rispens T. Immunoglobulin G4: an odd antibody. Clin Exp Allergy. 2009;39(4):469_ 77. https://doi.org/10.1111/j.1365-2222.2009.03207.x.

28. Partridge MA, Karayusuf EK, Dhulipala G, Dreyer R, Daly T, Sumner G, et al. Matrix interference from Fc-Fc interactions in immunoassays for detecting human IgG4 therapeutics. Bioanalysis. 2015;7(20):2701-12. https://doi.org/10.4155/ bio.15.152.

29. Partridge MA, Vijayam U, Karayusuf EK, Shum E, Sirimanne $\mathrm{T}$, Garlits $\mathrm{J}$, et al. Bridging immunogenicity assays for IgG4 therapeutics: mitigating interference from Fc-Fc interactions. Bioanalysis. 2017;9(9):707-17. https://doi.org/10.4155/bio-20170011.

30. Tolbert WD, Subedi GP, Gohain N, Lewis GK, Patel KR, Barb AW, et al. From Rhesus macaque to human: structural evolutionary pathways for immunoglobulin $G$ subclasses. mAbs. 2019;11(4):709-24. https://doi.org/10.1080/19420862.2019.1589852.

31. Falkenburg WJ, van Schaardenburg D, Ooijevaar-de Heer P, Wolbink G, Rispens T. IgG subclass specificity discriminates restricted IgM rheumatoid factor responses from more mature anti-citrullinated protein antibody-associated or isotypeswitched IgA responses. Arthritis Rheum. 2015;67(12):312434. https://doi.org/10.1002/art.39299.

32. Falkenburg WJ, van Schaardenburg D, Ooijevaar-de Heer P, Tsang ASMW, Bultink IE, Voskuyl AE, et al. Anti-hinge antibodies recognize IgG subclass- and protease-restricted neoepitopes. J Immunol. 2017;198(1):82-93. https://doi.org/ 10.4049/jimmunol.1601096.

Publisher's Note Springer Nature remains neutral with regard to jurisdictional claims in published maps and institutional affiliations. 